\title{
Uterine Ligament
}

National Cancer Institute

\section{Source}

National Cancer Institute. Uterine Ligament. NCI Thesaurus. Code C61360.

Any of the eight ligaments pertaining to the uterus including the anterior, posterior,

lateral, sacro-uterine and round ligaments. 